\title{
Research on identification and countermeasures of the construction project team's Unethical Pro-Organizational Behavior
}

\author{
Yu Ji, Chunling Sun \\ School of Management, Tianjin University of Technology, Tianjin, 300384, China \\ jy_everyday@163.com
}

\begin{abstract}
Due to the uncertainty of external environment and internal incompleteness of contract, the project team members are, more often than not, prone to exhibit Unethical Pro-Organizational Behavior. This paper focus on the construction project team's Unethical Pro-Organizational Behavior. According to the former literature research of enterprise employee's Unethical Pro-Organizational Behavior to concluding the concept definition, characteristics and mechanism. Then identifying the construction project team's Unethical Pro-Organizational Behavior based on the quality, progress and cost target. Proposing preventive measures from three aspects, including the ethical atmosphere, the individual moral standards and the demonstration effect of leadership. The study intends to provide theoretical support for contractor to prevent the construction project team's Unethical Pro-Organizational Behavior.
\end{abstract}

Keywords: The construction project team members; Unethical Pro-Organizational Behavior; Identification; Countermeasures.

\section{Introduction}

In recent years, there are more unethical activities in our country, so the moral crisis has become the common anxiety of modern society. Ethical issues which have been extended to the outside of the scope of traditional philosophy, become the common issues for academia and work organizations [1]. Unethical Pro-Organizational Behavior (UPB) is intended to benefit organizations. Although UPB is a form of unethical behavior, it differs from many other forms in that it is voluntary, extra-role, and prosocial and is intended to be beneficial rather than harmful to organizations [2]. Its essential characteristics include two aspects: first, the act is illegal and immoral, which is unethical; second, the intention of the act is to benefit the organization or its members, and thus is pro-organized. Therefore, UPB is a kind of special unethical activities, because its pro-organized and easily overlooked, it also can be encouraged. But this behavior will not only hurt the interests of stakeholders, but also damage the long-term interests of enterprises. Under such social background, it is significant to research Unethical Pro-Organizational Behavior.

Construction project team is composed of the knowledge workers from different professional background and technical expertise to complete the construction project. The temporary team is belong to the contractor in the construction project management. Because the external environment of the construction industry is not controllable and the internal contract is incompleteness, the construction team members needs to play initiative and exceed job role to do organizational citizenship behavior for the success of construction project. But the project team members will focus more on the current interests of the organization inevitably, and easily neglect the long-term performance and sustainable development of the organization. So once UPB exists, it will not only cause huge losses to the contractor, but also involve other stakeholders, resulting in multiple effects, or even quality problems resulting in casualties. At present, there were few research on it, therefore, it is significant to explore UPB of the project team members in the Chinese context. It can also help to solve the problem of ethical management of the project team in the construction process. Based on this, the paper focus on the construction project team's Unethical Pro-Organizational Behavior and provide theoretical support for contractor to prevent the construction project team's Unethical Pro-Organizational Behavior. 


\section{Literature Review and Concept Definition}

\subsection{Literature Review of Unethical Pro-Organizational Behavior}

Moral research in organizations originated in the 1970s. Employee's workplace immorality is widespread in various social organizations at all levels, covering commercial organizations, government organizations, academic organizations and other organizations, and the organization's long-term performance and sustainable development of immeasurable damage [3]. However, Ashforth [4] and others study shows that individuals in addition to personal interests for the same time to make harm to the organization or its members of the unethical behavior, will also be conducive to others or organizations to make unethical behavior. Umphress et al. First proposed the concept of UPB in 2010, and defined it as the unethical behavior of employees to protect the interests of the organization [5].

In the past, empirical research on immoral pro-organizational behavior is not so much. Miao [6]and other people using the questionnaire survey to the government civil servants for the survey, starting from the moral leadership and recognition of the boss, the behavior of unethical behavior of the pro-factor factors were tested, the results show that moral leadership and subordinates of the immoral pro-organizational behavior there is inverted "U"-type curve. Effelsberg [7] and other people use the questionnaire survey method to the employees of the survey object, from the transformational leadership, organizational identity and moral (immoral) behavior of personal tendencies of the three aspects of the impact of unethical behavior of the pro-organizational factors Two empirical studies have shown that transformational leadership has a significant positive impact on the willingness of subordinate unorganized behavior.

\subsection{Definition of the Construction Project Team's Unethical Pro-Organizational}

Because the external environment of the construction industry is not controllable and the internal contract is incompleteness, the construction team members needs to play initiative and exceed job role to do organizational citizenship behavior for the success of construction project. Although for the purpose of safeguarding the interests of the organization made, may allow enterprises to temporarily profit, but because of this violation of ethical standards, it must not have sustainability. The project caused huge losses, while affecting the image of contractors. For example, because the contractor in the tender offer reported a low price, resulting in little profit or even unprofitable situation, it will jerry in the construction process, the procurement of materials is also shoddy; contractors and design units conspiracy, The contractor and the owner conspiracy in order to seek the interests of the contractor and the owner conspiracy to seek the interests of the contractor and the supervision unit conspiracy and so on.

In order to have a deeper understanding of the connotation of the immoral pro-organizational behavior of the project team, the characteristics of the project team are analyzed: (1) the project team members are conscious spontaneous behavior;(2) intend to improve the performance; (3)compliance with organizational expectations, organization and leadership acquiescence; (4) in the short term does enhance the corporate earnings, but the performance of the purpose; (5) in the formal description of the job description does not have the order of the supervisor; From a long-term point of view has caused adverse effects, damage to the interests of enterprises, undermine the corporate image.

\section{Identification of the Construction Project Team's Unethical Pro-Organizational Behavior}

The main stakeholders in the construction process include the owners and contractors, and the secondary stakeholders include the supervision unit and the design unit. According to information economics research on asymmetric information, there are often more information asymmetry in the construction process.

By analyzing the characteristics of the construction phase, the task type, the risk control and the stakeholders involved, the characteristics of the construction tasks can be divided into three dimensions: (1) the quality of construction; (2) the construction cost ; (3) construction progress. The three characteristics of the construction process are the important factors considered by the owners, 
contractors, and other stakeholders, such as the supervision and design units, in the project-related decision-making. According to the three characteristics of the construction process, the project team can divide the work tasks in the construction process. On this basis, it can identify the unethical pro-organization behavior that the project team members may make during the construction process.

As can be seen from the before, the construction process of the project team members of the immoral pro-organizational behavior mainly for the hidden actions of moral hazard and hidden moral hazard information. Team members act as an agent after signing a contract (choose whether to construct according to the contract), or choose actions in a given natural state (choose whether to report the actual status of the construction phase to the owner). Because of the asymmetry of information, uncertainties and incompleteness of contract, team members tend to choose to maintain the interests of contractors, thus making immoral pro-organizational behavior.

\section{Countermeasures of the Construction Project Team's Unethical Pro-Organizational Behavior}

Due to the uncontrollability of the external environment and the incompleteness of the internal contract, the project team members often need to show more pro-organization in the process of construction. But in this process, the project team members tend to focus too much on the maintenance of organizational interests and promote the effective operation of the organization, it is easy to cause unethical behavior of the organization. Therefore, it is necessary to take reasonable measures to avoid the immoral behavior of the project team members.

(1) Improve the ethical atmosphere of the project team. In the game between the contractor and the owner, the contractor is in a relatively inferior position, so it is more inclined to pursue the maximization of interests through other means. Most of the team members are in the customary level of moral cognition development, so their work behavior is more inclined to be affected by the internal system and team culture. Under the influence of egoism and interest-driven team atmosphere, the team members will show more unethical pro-organizational behavior, thus polluting the moral culture atmosphere within the project team, leading to the unethical behavior of the team and spread. The contractor should establish the corresponding reward and punishment system and perfect moral incentive mechanism. The ethical atmosphere within the project team should be influenced by the scientific and reasonable moral regulations and the healthy and healthy moral culture environment, and cultivate the correct moral values of the team members.

(2) Improve the project team members individual moral standards. The moral and internal factors of the project team members are the main reasons of UPB, and the construction of the project is also completed by the members of the project team. However, in the current construction process, there is often a low level of morality of the project team members. Therefore, the contractor should consider the level of moral development of the project team in the formation of the project team, and select members with high moral identity. In addition, the contractor should strengthen the ethical training of project team members to promote their ethical behavior. First of all, moral training can convey the contractor's attention to moral information; Secondly, through training can make the team members more clearly aware of the causes of immoral pro-organization behavior and the possible serious consequences, so that in the face of ethical dilemma, Can make a rational decision-making for the quality of the project and all relevant stakeholders.

(3) Improve the demonstration effect of the leadership. If the leadership of the project team is the promoter of unethical pro-organizational behavior, then the team members will be more inclined to follow and imitate them, leading to the entire team from top to bottom spread of unethical pro-organizational behavior. It can be seen that the team atmosphere created by team leadership is usually the basis of organizational ethical culture, and if team leaders behave improperly, the team members' perceptions of the results of unethical pro-organizational behavior will be distorted. Therefore, the contractor as managers to unscrupulous pro-organizational behavior to strengthen the management of the behavior of the immoral behavior of the harm to have a clear understanding, not 
because of its short-term success in favor of the project to encourage team members to do the kind of behavior Ignoring its long-term hazards. In addition, the contractor shall establish relevant policies and regulations to restrict the behavior of unscrupulous pro-organizations and strengthen the education and management of project team members, and shall not connive at or tolerate unethical pro-organizational behavior of project team members.

\section{Summary}

This paper focus on the construction project team's Unethical Pro-Organizational Behavior. According to the former literature research of enterprise employee's Unethical Pro-Organizational Behavior to concluding the concept definition, characteristics and mechanism. Then identifying the construction project team's Unethical Pro-Organizational Behavior based on the quality, progress and cost target. Proposing preventive measures from three aspects, including the ethical atmosphere, the individual moral standards and the demonstration effect of leadership. The study intends to provide theoretical support for contractor to prevent the construction project team's Unethical Pro-Organizational Behavior. The future research needs to be further deepened, and quantitative methods should be used in combination with qualitative research to enhance the persuasiveness of the research, so as to provide a basis for the management of constructors.

\section{References}

[1] Kish-Gephart, J.J., Harrison, et al. Meta-Analytic Evidence about Sources of Unethical Decisions at Work. Journal of Applied Psychology, Vol.95 (2010) No.1, p.1-31.

[2] Umphress E. E., Bingham J. B. When Employees Do Bad Things for Good Reasons: Examining Unethical Pro-Organizational Behaviors. Organization Science, Vol.22 (2011) No3, p.621-640.

[3] Lavelle J J, Brockner J, Konovsky M A, et al. Commitment, procedural fairness, and organizational citizenship behavior: A multifocal analysis. Journal of Organizational Behavior, Vol.30 (2009) No7, p.337-357.

[4] Ashforth, B.E., \& Anand, V. The normalization of corruption in organizations. Research in Organizational Behavior, Vol.25(2003) p.1-52.

[5] Umphress E. E., Bingham J. B., Marie S. M. Unethical Behavior in the Name of the Company: The Moderating Effect of Organizational Identification. Journal of Applied Psychology, Vol.95 (2010) No4, p.769-780.

[6] Miao, Q., Newman, A., Yu, J., \& Xu, L. The relationship between ethical leadership and unethical pro-organizational behavior: Linear or curvilinear effects? Journal of Business Ethics, Vol.30 (2013) No116, p.641-653.

[7] Effelsberg, D., Solga, M. \& Gurt, J. Transformational leadership and follower's unethical behavior for the benefit of the company: A two-study investigation. Journal of Business Ethics, 2013, p. 1-13. 